\title{
Prediction of outcome in dilated cardiomyopathy
}

\author{
RIENZI A DIAZ, AUSTINE OBASOHAN, CELIA M OAKLEY \\ From the Department of Medicine (Clinical Cardiology), Royal Postgraduate Medical School, Hammersmith \\ Hospital, London
}

SUMMARY One hundred and sixty nine patients (mean age $39 \cdot 1$ years) with documented dilated cardiomyopathy were studied for a mean of 5.5 years One hundred and four patients died during this period. The average (SD) interval from the onset of symptoms to death was 4.1 (3.7) years. One year and five year mortality rates were $27.8 \%$ and $57.4 \%$ respectively. Most of the deaths occurred within two years of diagnosis. The only difference between survivors and those who died was in the severity of left ventricular dysfunction at the time of referral. Significant differences between survivors and non-survivors were found for left ventricular end diastolic pressure (17.3 versus $23.4 \mathrm{~mm} \mathrm{Hg}$ ), left ventricular end systolic volume (87.4 versus $\left.128.9 \mathrm{ml} / \mathrm{m}^{2}\right)$, left ventricular end diastolic volume $\left(130.7\right.$ versus $\left.173.2 \mathrm{ml} / \mathrm{m}^{2}\right)$, and ejection fraction ( 32.8 versus $25.4 \%$ ). The duration of previous symptoms, preceding virus infection, positive family history, recent pregnancy, or heavy alcohol intake did not seem to influence prognosis. Nor did treatment, which was similar in both groups with a quarter of the patients receiving vasodilators.

Patients with dilated cardiomyopathy have a high mortality irrespective of treatment. The only identifiable prognostic indicator was the severity of left ventricular impairment at referral.

Dilated cardiomyopathy is a heart muscle disease of unknown cause in which one or both ventricles are dilated and poorly contracting. ${ }^{1-3}$ Various clinical, radiological, angiographic, haemodynamic, and histopathological variables have been suggested as predictors of outcome in dilated cardiomyopathy, but there is no uniform agreement about their usefulness. ${ }^{4-6}$

One year mortality in this condition may vary from $31 \%$ to $35 \%{ }^{467}$ and five year mortality has been reported to be around $50 \%{ }^{7-9}$ This poor prognosis seems not to have been modified during the past two decades, despite the introduction of new diuretics and the use of vasodilators and anticoagulants during this period.

To strengthen understanding of the clinical course of this disorder we report experience at Hammersmith Hospital in the diagnosis, treatment, and follow up of these patients. To our knowledge this is the largest series of patients with well documented dilated cardiomyopathy so far reported and the observations are relevant to the selection of patients

Requests for reprints to Dr Celia M Oakley, Department of Clinical Cardiology, Hammersmith Hospital, Du Cane Road, London W120HS.

Accepted for publication 1 June 1987 for cardiac transplantation and the timing of the operation.

\section{Patients and methods}

We retrospectively analysed data on 209 consecutive patients with dilated cardiomyopathy who were first seen in the Clinical Cardiology Unit (Department of Medicine) at Hammersmith Hospital from March 1962 to March 1982. Only 169 patients were included in the study. Eighteen patients were excluded because data were incomplete and twenty two were excluded because they had predominant or exclusive dilatation of the right ventricle. Most patients were referred for the evaluation of cardiac failure. All patients were followed up in the outpatient department. The follow up period was defined as the interval from the date of first admission to Hammersmith Hospital until either death or 31 March 1983. The interval from the onset of symptoms to the first admission was also analysed. There were 131 men and 38 women. One hundred and thirty eight patients were European, 17 were black, and 14 were Asian.

Dilated cardiomyopathy was identified as a heart muscle disease of unknown cause with dilatation of the left or right heart or both ${ }^{1-3}$; so the diagnosis 
was made by exclusion of other causes of cardiac failure. Exclusion criteria included: heart muscle disease of known origin (for example amyloid, sarcoid, haemochromatosis), clinical or angiographic coronary heart disease or both (patients with obstruction of $\geqslant 50 \%$ of one or more major coronary artery branches or who had considerable irregularity of one or more vessels were excluded even if it was thought that the left ventricular failure could not be attributed to the coronary disease shown on angiography), systemic hypertension, and hypertrophic cardiomyopathy. The diagnosis required a left ventricular ejection fraction of $<45 \%$ and usually included also: (a) a history of dyspnoea on exertion or at rest; $(b)$ physical signs of left or right heart failure or both; (c) cardiomegaly on chest $x$ ray; (d) one or more of the following electrocardiographic abnormalities-atrial fibrillation or atrial flutter, conduction system disturbances, and evidence of a left ventricular disorder usually with high voltage in the chest leads and repolarisation abnormalities; (e) left ventricular end diastolic pressure $>15 \mathrm{~mm} \mathrm{Hg}$.

The symptomatic state was classified according to the New York Heart Association classification. Death was defined as sudden when it was unexpected and occurred within 24 hours of the onset of new symptoms. Complex ventricular arrhythmia was defined as the presence of multiform or repetitive ventricular extrasystoles (couplets or bursts of ventricular tachycardia) or both.

To evaluate possible risk factors we analysed alcohol intake, recent pregnancy, family history and febrile illness heralding the onset of symptoms, and positive anti-viral titres.

\section{MEASUREMENTS}

All patients had a baseline 12 lead electrocardiogram and chest $x$ ray. $\mathrm{R}$ waves in the left precordial lead V5 over $20 \mathrm{mV}$ were considered as high voltage and under $5 \mathrm{mV}$ as low voltage. Ambulant electrocardiogram monitoring was carried out in all patients seen after $1980-57 \%$ of the total series. Blood samples were also taken to measure serum iron, antinuclear factor, thyroid function, rheumatoid factor, and virus antibody titres.

HAEMODYNAMIC AND ANGIOGRAPHIC STUDIES All patients underwent cardiac catheterisation by the percutaneous approach through the femoral or brachial artery. Left and right heart pressures were recorded with an Electronics for Medicine DR8 recorder. Fluid filled lines and P23D Statham transducers were used. The mid chest was used as a reference point for the calibration of zero.

Left ventricular volumes were determined by
Grant et al's method ${ }^{10}$ from the biplane anteroposterior and lateral projections filmed at 30 frames/s after the injection of contrast material into the left ventricle. The ejection fraction (EF) was calculated by the formula: $\mathrm{EF}=$ (LVEDV LVESV)/LVEDV, where LVESV = left ventricular end systolic volume and LVEDV = left ventricular end diastolic volume. Volumes are given as millilitres per square metre of body surface area $\left(\mathrm{ml} / \mathrm{m}^{2}\right)$. Ninety seven $(57 \%)$ of the patients underwent coronary angiography. This includes all patients studied since 1980 irrespective of age. Those who did not have angiography had no clinical, electrocardiographic, or ventriculographic features of coronary heart disease and none who came to necropsy had clinically significant coronary atheroma. Thirty two $(18.8 \%)$ patients underwent percutaneous right ventricular endomyocardial biopsy. Biopsy was used to identify myocarditis and the specific heart muscle diseases. ${ }^{11}$ All endomyocardial biopsy specimens in the current series showed nonspecific morphological abnormalities and there was no evidence of active or past myocarditis. Since March 1983 we have performed myocardial biopsies on 39 more patients with clinically similar dilated cardiomyopathy, 21 of whom were reported as showing features of active past or healing myocarditis on biopsy.

Echocardiographic and radionuclide measurements were not included in this analysis because they only became available after the series was started.



Fig 110 year survival curve of 169 patients with dilated cardiomyopathy. Numbers in parentheses are patients alive at the beginning of interval. Numbers at years 6, 7, 8, and 9 were omitted because of the small amount of attrition between 5 and 10 years. 


\section{TREATMENT}

All patients were taking diuretics. Digitalis preparations were not used routinely in patients in sinus rhythm. Anticoagulant drugs are normally prescribed when dilated cardiomyopathy is diagnosed, irrespective of the cardiac rhythm. One quarter of the patients received vasodilator drugs.

\section{STATISTICAL ANALYSIS}

The data were stored in a computer and analysed by the statistical package, Minitab. The probability of survival from the time of first evaluation at Hammersmith Hospital was analysed by the life table method. The $t$ test and $\chi^{2}$ test were used to analyse significance of univariate prognostic factors. Linear regression analysis was carried out to test the relation between clinical or haemodynamic variables and the duration of survival in those who died. Results were considered to be statistically significant when $\mathrm{p}<0.05$

\section{Results}

One hundred and sixty nine patients with documented dilated cardiomyopathy were studied. The mean (SD) age at the onset of symptoms was $39 \cdot 1(14 \cdot 1)$ years. The interval from the onset of symptoms to first evaluation at Hammersmith Hospital was 1.8 (2.8) years. The average duration from the onset of symptoms to death was $4 \cdot 1$ ( 3.7 years). The mean follow up period was $5.5(4.2)$ years. Patients who survived five years were unlikely to die thereafter (fig 1).

\section{CLINICAL CHARACTERISTICS}

Table 1 summarises the clinical symptoms and physical signs found on the first admission to Hammersmith Hospital. The commonest symptom was dyspnoea either on exertion or at rest $(85.8 \%)$ followed by palpitation and oedema. Chest pain was not uncommon and it was present in 35 patients $(20.7 \%)$, all of whom underwent coronary angiography which excluded clinically significant coronary artery disease as defined earlier. A mitral regurgitant murmur was present in $37.9 \%$ of the patients. Pulmonary or systemic embolism had occurred in $10.6 \%$ and $8.3 \%$ respectively before referral to Hammersmith. Atrial fibrillation was present in only $23.7 \%$ of those patients who had had embolic events. Asymptomatic cardiomegaly was the first manifestation of illness in eight $(4.7 \%)$ patients.

All patients had radiographic evidence of cardiomegaly and the average cardiothoracic index was $58 \cdot 8(6 \cdot 8 \%)$.

Table 2 shows electrocardiographic features. Atrial fibrillation and ventricular extrasystoles were
Table 1 Clinical characteristics of patients with dilated cardiomyopathy

\begin{tabular}{lrc}
\hline Feature & No & $\%$ \\
\hline Dyspnoea & 145 & $85 \cdot 8$ \\
Third heart sound & 145 & 85.8 \\
MR murmur & 64 & 37.9 \\
Palpitation & 50 & $29 \cdot 6$ \\
Oedema & 49 & 28.9 \\
Chest pain & 35 & 20.7 \\
Pulmonary emboli & 17 & 10.6 \\
Systemic emboli & 14 & 8.3 \\
\hline
\end{tabular}

MR, mitral regurgitation

Table 2 Electrocardiographic features of patients with dilated cardiomyopathy

\begin{tabular}{lcc}
\hline Feature & No & $\%$ \\
\hline Sinus rhythm & 129 & $76 \cdot 3$ \\
Atrial fibrillation & 34 & $20 \cdot 1$ \\
LBBB & 35 & $20 \cdot 7$ \\
RBBB & 7 & $4 \cdot 1$ \\
VE isolated & 43 & $25 \cdot 4$ \\
VE complex & 25 & $14 \cdot 8$ \\
High voltage V5 & 54 & $34 \cdot 4$ \\
Low voltage V5 & 5 & $3 \cdot 2$ \\
LA enlargement & 76 & $44 \cdot 9$
\end{tabular}

LBBB, left bundle branch block; RBBB, right bundle branch block; VE, ventricular extrasystoles; LA, left atrium.

the commonest arrhythmias present in this series. Complex ventricular arrhythmias were present in $14.8 \%$ of the patients. Complete left bundle branch block was the commonest conduction system defect $(20.7 \%)$. Right bundle branch block was rarely present $(4 \cdot 1 \%)$. High voltages $(>20 \mathrm{mV})$ in the left precordial lead V5 occurred in $34.4 \%$ of the cases while low voltages $(<5 \mathrm{mV})$ were occasionally seen $(3 \cdot 2 \%)$. Arrhythmias were documented by routine Holter recording in 97 patients investigated after 1980. Before that date, complex ventricular arrhythmias or ventricular tachycardia were only found by chance recording or when they caused symptoms.

\section{HAEMODYNAMIC FEATURES}

Table 3 shows the baseline haemodynamic findings. The left ventricular end diastolic pressure was raised $(20.8(9.8) \mathrm{mm} \mathrm{Hg})$ and the ejection fraction was reduced $(29 \cdot 2(12 \cdot 7) \%)$. Both the left ventricular end systolic volume $\left(104 \cdot 7(57 \cdot 1) \mathrm{ml} / \mathrm{m}^{2}\right)$ and the end diastolic volume $\left(148.4(64.2) \mathrm{ml} / \mathrm{m}^{2}\right)$ were increased. The mean stroke volume (42.1 (22.5) $\mathrm{ml} / \mathrm{m}^{2}$ ) was at the lower limit of normal but there was wide variation.

The left ventricular angiograms showed a markedly dilated and poorly contracting left ventricle in all patients, with mitral regurgitation ranging from 0 to moderate (grade 2 out of 4 ).

During the period of analysis 104 of the $169 \mathrm{pa}-$ tients died. Nearly all deaths were cardiac in origin 
Table 3 Haemodynamic and angiographic variables of patients with dilated cardiomyopathy

\begin{tabular}{lrl}
\hline Variable & Mean $(S D)$ & Normal value \\
\hline LVEDP (mm Hg) & $20 \cdot 8(9 \cdot 8)$ & $<12$ \\
LVESV $\left(\mathrm{ml} / \mathrm{m}^{2}\right)$ & $104 \cdot 7(57 \cdot 1)$ & $21-34$ \\
LVEDV $\left(\mathrm{ml} / \mathrm{m}^{2}\right)$ & $148 \cdot 4(64 \cdot 2)$ & $64-96$ \\
SV $\left(\mathrm{ml} / \mathrm{m}^{2}\right)$ & $42 \cdot 1(22 \cdot 5)$ & $43-62$ \\
EF $(\%)$ & $29 \cdot 2(12 \cdot 7)$ & $63-72$ \\
\hline
\end{tabular}

LVEDP, left ventricular end diastolic pressure; LVESV, left ventricular end systolic volume; LVEDV, left ventricular end diastolic volume; SV, stroke volume; EF, ejection fraction.

and sudden death occurred in $8.6 \%$ of the cases. Figure 1 shows the cumulative survival rates. The survival curve shows a one year survival rate of $72 \cdot 2 \%$. Five year survival was $42 \cdot 6 \%$. Most of the deaths occurred during the first two years of follow up $(41.4 \%)$.

In order to determine whether there were differences between patients who were still alive (survivors) and those who were dead (non-survivors) at the end of the analysis period we compared the baseline clinical, serological (virus), electrocardiographic, radiographic, and haemodynamic variables of both groups. No significance differences were found either in the clinical or in the electrocardiographic variables between survivors and nonsurvivors nor any difference in the cardiothoracic ratio (table 4). The only significant differences between survivors and non-survivors were with the haemodynamic and angiographic variables shown in table 5. Those who died had higher left ventricular end diastolic pressure than did the survivors (23.4 versus $17.3 \mathrm{~mm} \mathrm{Hg} ; \mathrm{p}<0.001)$. They also had lower ejection fractions $(25.4$ versus $32.8 \% ; p<0.005)$ and larger left ventricular end systolic volumes $\left(128.9\right.$ versus $\left.87.4 \mathrm{ml} / \mathrm{m}^{2} ; \mathrm{p}<0.01\right)$ and left ventricular end diastolic volumes $(173.2$ versus $130.7 \mathrm{ml} / \mathrm{m}^{2} ; \mathrm{p}<0.01$ ) than did the survivors. The stroke volume was similar in both groups (43.2 versus $40.6 \mathrm{ml} / \mathrm{m}^{2} ; \mathrm{p} \mathrm{NS}$ ).

Linear regression analysis between the clinical, electrocardiographic, radiographic, haemodynamic,

Table 4 Comparison of clinical, electrocardiographic, and radiological variables (mean $(S D)$ ) in survivors and those who died

\begin{tabular}{llll}
\hline & Survivors & Died & $p$ \\
\hline Patients (No) & 67 & 102 & NS \\
Age (yr) & $42 \cdot 4(13 \cdot 1)$ & $37 \cdot 0(14 \cdot 4)$ & NS \\
LA enlargement (mm) & $55 \cdot 7(7 \cdot 2)$ & $57 \cdot 9(6 \cdot 2)$ & NS \\
Atrial fibrillation (No) & $28 / 67$ & $48 / 102$ & NS \\
VPB complex (No) & $16 / 67$ & $18 / 102$ & NS \\
LBBB (No) & $14 / 67$ & $22 / 102$ & NS \\
Embolism (No) & $10 / 67$ & $21 / 102$ & NS \\
\hline
\end{tabular}

See table 2 for abbreviations.

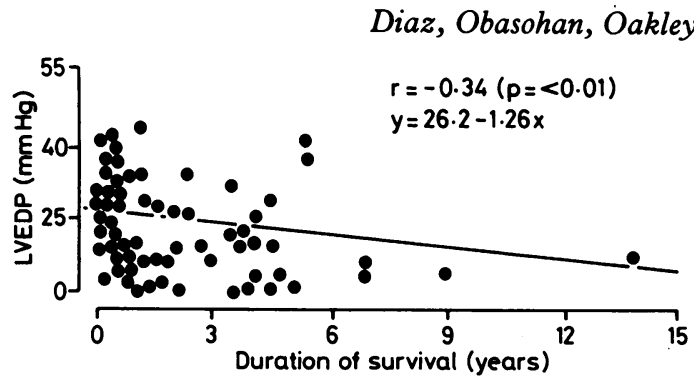

Fig 2 Relation between left ventricular end diastolic pressure (LVEDP) and duration of survival in 65 patients who died. Most patients died within three years of referral and most of those who survived longer had lower LVEDP, although there are notable exceptions. (There is no linear correlation between the value of LVEDP and duration of survival.)

and angiographic variables and the length of survival in those who died showed a relation only with left ventricular end diastolic pressure, which was inversely correlated with duration of survival (fig 2).

Furthermore, we also compared 24 patients $(14.2 \%)$ in whom the first manifestation of illness was not cardiac failure (for example arrhythmias, embolism, asymptomatic cardiomegaly) with 145 patients $(85.8 \%)$ in whom cardiac failure was the mode of presentation. There were no significant differences in mortality and length of survival (table 6).

The presence of "risk factors" such as family history, recent pregnancy, preceding virus infection, or heavy alcohol consumption did not seem to influence prognosis. All patients were advised against taking alcohol but the effect on left ventricular function and prognosis of stopping drinking could not be analysed because there were no apparent or admitted heavy drinkers in the series who could be shown to have stopped drinking and no dramatic improvements among them. At the last follow up visit the symptomatic state according to the New York Heart Association classification was significantly better in survivors than it was in non-survivors (table 7). The clinical state had deteriorated before death in half of those who died $(47.6 \%)$ compared with only $14 \%$ of

Table 5 Comparison of haemodynamic and angiographic variables (Mean (SD)) in survivors and those who died

\begin{tabular}{lccc}
\hline Variable & Survived & Died & $p$ \\
\hline LVEDP (mm Hg) & $17.3(7.8)$ & $23.4(9.3)$ & $<0.001$ \\
LVESV (ml/m $\left.\mathrm{m}^{2}\right)$ & $87.4(58.6)$ & $128.9(45.8)$ & $<0.01$ \\
LVEDV $\left(\mathrm{ml} / \mathrm{m}^{2}\right)$ & $130.7(66.7)$ & $173.2(52.9)$ & $<0.01$ \\
EF $(\%)$ & $32.8(11.6)$ & $25.4(12.8)$ & $<0.005$ \\
\hline
\end{tabular}

See table 3 for abbreviations. 
Table 6 Duration of survival and number of deaths according to first manifestation of illness in 169 patients with dilated cardiomyopathy

\begin{tabular}{llll}
\hline & Heart failure & Other & $p$ \\
\hline Deaths (No) & $88 / 145$ & $14 / 24$ & NS \\
Survival (yr) & $2 \cdot 2(2 \cdot 4)$ & $2 \cdot 6(3 \cdot 8)$ & NS \\
\hline
\end{tabular}

Table 7 Symptomatic state at the last follow up

\begin{tabular}{lll}
\hline NYHA & Survivors & Non-survivors \\
\hline Class I or II & $85 \cdot 2 \%$ & $30 \cdot 7 \%$ \\
Class II or IV & $14 \cdot 2 \%$ & $69 \cdot 3 \%$
\end{tabular}

NYHA, New York Heart Association classification.

Table 8 Vasodilator treatment

\begin{tabular}{lccl}
\hline Variable & No vasodilators & Vasodilators & $p$ \\
\hline LVEDP $(\mathrm{mm} \mathrm{Hg})$ & $19.5(8.9)$ & $22.5(9 \cdot 6)$ & NS \\
LVEDV $\left(\mathrm{ml} / \mathrm{m}^{2}\right)$ & $135.8(53.0)$ & $167.8(84 \cdot 8)$ & NS \\
LVESV $\left(\mathrm{ml} / \mathrm{m}^{2}\right)$ & $92.1(41.6)$ & $126.2(82 \cdot 0)$ & NS \\
EF $(\%)$ & $30.2(13.2)$ & $26.9(12 \cdot 3)$ & NS \\
\hline
\end{tabular}

See table 3 for abbreviations.

the survivors $-86 \%$ of the survivors were either stable or had improved their functional state. Death in $52.4 \%$ of the non-survivors was unexpected because their clinical state had not deteriorated.

All patients were taking diuretics and those in atrial fibrillation were taking digoxin. Vasodilators had been prescribed to one quarter of the patients. Patients given vasodilators had larger left ventricular end systolic and end diastolic volumes, and higher end diastolic and lower ejection fractions than those not receiving vasodilator treatment-but these differences were not significant (table 8).

\section{Discussion}

Dilated cardiomyopathy is a heart muscle disease of unknown cause..$^{1-3}$ All four cardiac chambers may be dilated ${ }^{1213}$ or only the left ventricle, ${ }^{314}$ and rarely only the right ventricle is dilated. ${ }^{15}$ Patients with only right ventricular dilatation were not included in this study. The commonest mode of presentation is heart failure. ${ }^{5916}$ Both shortness of breath and ventricular gallop were present in $85.8 \%$ of our patients. The clinical course is unpredictable. Most of these patients worsened quickly and died within the first two years after referral and within four years of the onset of symptoms, irrespective of the treatment used. Because we are a tertiary referral centre these figures may seem to be worse than they really are. Some of our patients referred after one to two years of treatment were undoubtedly only referred because they were deteriorating. Patients who were "doing all right", overt alcoholics, or the elderly may not have been referred. Despite this probable selection, a trend towards rapid mortality has been reported in other published series as well as ours $^{457}$ and the prognosis in this heart condition is one of the poorest. In the current series we observed a one year mortality of $27.8 \%$ and a five year mortality of over $50 \%$. Most of our patients died within two years of the first evaluation at Hammersmith Hospital (fig 1). Conversely, patients who survived longer than this showed little tendency to die later. These were the patients who came to us despite not being on an obviously down-hill course. Their duration of symptoms before referral was similar to that in the patients who died. It is possible, indeed probable, that we are dealing with more than one disease process, but it is important to realise that a few patients with dilated cardiomyopathy can do well, with a good quality of life, and stable condition over many years even if they do not actually improve or "get better".

In several reports some clinical and haemodynamic variables have been advocated as useful predictors of the clinical course. ${ }^{17-19}$ Years ago Hatle et al reported a poor outcome in those patients with both low voltages and evidence of left atrial enlargement on the electrocardiogram. ${ }^{18}$ On the other hand, atrial fibrillation and left ventricular hypertrophy are factors considered to be associated with longevity. ${ }^{68}$ Though the survivors in the current series showed a trend towards higher voltage on the electrocardiogram than those who died, the difference was not significant nor was the difference in the presence of atrial fibrillation. A favourable course has also been reported in patients who developed systemic hypertension after successful treatment for heart failure. ${ }^{18}$ Patients in whom blood pressure rises while they are being treated for heart failure are likely to have hypertension rather than cardiomyopathy ${ }^{3}$ and when this feature was seen in our patients we diagnosed hypertension. Recently Fuster $e t$ al described four factors which had predictive value in their series. ${ }^{7}$ These were age $>55$ years, cardiothoracic ratio $>0.55$, cardiac index $<$ $3.01 / \mathrm{min} / \mathrm{m}^{2}$, and left ventricular end diastolic pressure $>20 \mathrm{~mm} \mathrm{Hg}$. The presence of ventricular arrhythmias and conduction system defects on the electrocardiogram has been reported to be associated with bad prognosis in dilated cardiomyopathy. ${ }^{19} 20$ Others did not observe such an association ${ }^{21}$ nor did we in this series. Recently two studies of ventricular arrhythmias were carried out in patients with dilated cardiomyopathy by means of long term ambulatory 
electrocardiogram monitoring. ${ }^{22} 23$ Huang et al reported that ventricular tachycardia was not predictive in their series. ${ }^{22}$ Von Olshausen et al also concluded that patients at higher risk are more reliably identified by their severely impaired left ventricular function than by ventricular arrhythmias. ${ }^{23}$ This fits in with our observation that death was rarely unexpected and rarely occurred in patients with better preserved left ventricular function. Ventricular arrhythmias were most common in those with the poorest ventricular function. In our series the only significant differences between survivors and those who died applied to haemodynamic variables. Those who died had higher left ventricular end diastolic pressure, larger end systolic and end diastolic volumes, and lower ejection fractions than the survivors; and left ventricular function overrode all other features in the prediction of prognosis (table 5). This accords with previous reports. ${ }^{71724}$ Only end diastolic pressure showed significant inverse correlation with length of survival. The higher the end diastolic pressure, the shorter the survival period tended to be (fig 2). It is interesting and important that stroke volume was not predictive. The resting cardiac output was often normal, although it failed to rise normally on effort. This was associated with a less than normal rise in rate but the poor exercise response can only be explained by a fall in stroke volume on exercise even though ventricular filling is completed rapidly. This indicates a reduciton in filling, perhaps caused by a poorer systolic contraction on exercise or a decrease in compliance. Only after a very advanced stage does the resting cardiac output fall and this is shown by Fuster's bad prognostic factors one of which was a cardiac index of $<3.0$, which exceeds the lower limit of normal of most laboratories.

We did not find any correlation between prognosis and febrile illness heralding the onset of symptoms, pregnancy, or excessive alcohol intake. High neutralisation titres to Coxsackie B virus were similar in survivors and those who died but in a previous report from our institution an increased frequency of high titres had been found in patients with a short history of symptoms. ${ }^{25}$ Pulmonary and systemic embolism occurred in $10.1 \%$ and $8.3 \%$ respectively and all episodes occurred before referral to us and before the introduction of anticoagulants. These episodes had occurred irrespective of the cardiac rhythm. In fact, atrial fibrillation was only present in $23.7 \%$ of those patients who developed embolism. All patients with dilated cardiomyopathy seen at Hammersmith Hospital were given anticoagulants on diagnosis and no further embolism occurred.

All patients were on diuretics and all of those in atrial fibrillation were taking digoxin. One quarter of them were also taking vasodilator drugs. The efficacy of vasodilators in improving the haemodynamic state in these patients has already been proved ${ }^{26}{ }^{27}$ but the improvement in measured exercise capacity is disappointing. Since vasodilators tended to be given to patients with worse left ventricular function (table 8) it was not possible to evaluate statistically the effect of these drugs on prognosis but there was certainly no obvious benefit in patients with advanced disease.

Despite two decades of hard investigation of dilated cardiomyopathy, the underlying cause or causes are still unknown and no treatment is yet available to reverse pathogenesis. Only an understanding of the underlying causes will make specific treatment possible. Improvement in the prognosis of these patients will only come when this knowledge is coupled with earlier recognition. We were unable to confirm from our data the suggestion that virus myocarditis had preceded or initiated dilated cardiomyopathy but we are perplexed by the great increase in biopsy diagnosis of myocarditis since 1982 .

This is the largest series of patients with dilated cardiomyopathy so far reported. Its analysis confirmed that the disorder has a high mortality irrespective of the type of treatment, and the severity of left ventricular functional impairment (at referral) appears to be the main determinant of reduced survival, possibly because by the time symptoms develop or become at all limiting, dilatation may already have reached the point at which further deterioration is inevitable. Our observations are of importance in deciding the timing of cardiac transplantation. Some patients continue to do well and are stable for many years but patients with ejection fractions below $25 \%$ and deteriorating symptoms are unlikely to survive more than two years and may die at any time.

\section{References}

1 Oakley CM. Clinical definitions and classification of the cardiomyopathies. Postgrad Med J 1972;48:703-13.

2 Goodwin JF, Oakley CM. The cardiomyopathies. $\mathrm{Br}$ Heart $J$ 1972;34:545-52.

3 Oakley CM. Diagnosis and natural history of congestive (dilated) cardiomyopathy. Postgrad Med J 1978; 54:440-7.

4 Franciosa JA, Wilen M, Ziesche S, Cohn JN. Survival in men with severe chronic left ventricular failure due to either coronary heart disease or idiopathic dilated cardiomyopathy. Am J Cardiol 1983;51:831-6.

5 Segal JP, Stapleton JF, McClellan JR, Waller BF, Harvey WP. Idiopathic cardiomyopathy: clinical features, prognosis, and therapy. Curr Probl Cardiol 1978;3:1-48.

$6 \mathrm{Kuhn} \mathrm{H}$. Prognosis and possible presymptomatic manifestations of congestive cardiomyopathy. Postgrad Med J 1978;54:451-9. 
7 Fuster V, Gersh BJ, Giuliani ER, Tajik AJ, Branderburg RO, Frye RL. The natural history of idiopathic dilated cardiomyopathy. Am J Cardiol 1981;47:525-31.

8 Convert G, Delaye J, Beaune J, Biron A, Gonin A. Etude pronostique des myocardiopathies primitives non obstructives. Arch Mal Coeur 1980;3:227-37.

9 Kristinsson A. Diagnosis, natural history, and treatment of congestive cardiomyopathy. University of London, 1969:188. PhD Thesis.

10 Grant C, Raphael MJ, Steiner RE, Goodwin JF. Left ventricular volume and hypertrophy in outflow obstruction. Cardiovasc Res 1968;4:346-55.

11 Parrillo JE, Aretz HT, Palacios I, Fallon JT, Block PC. The results of tranvenous endomyocardial biopsy can frequently be used to diagnose myocardial diseases in patients with idiopathic heart failure: endomyocardial biopsies in 100 consecutive patients revealed a substantial incidence of myocarditis. Circulation 1984;69:93-101.

12 Roberts WC, Ferrans VJ. Pathologic anatomy of the cardiomyopathies: idiopathic dilated and hypertrophic types, infiltrative types, and endomyocardial disease with and without eosinophilia. Hum Pathol 1975;6:287-342.

13 Olsen ECG. Pathology of primary cardiomyopathies. Postgrad Med J 1972;48:732-7.

14 Croxson R, Raphael MJ. Angiographic assessment of congestive cardiomyopathy [Abstract]. $\mathrm{Br}$ Heart $\mathrm{J}$ 1969;31:390.

15 Fitchett DH, Sugrue DD, MacArthur CG, Oakley CM. Right ventricular dilated cardiomyopathy. $\mathrm{Br}$ Heart $J$ 1984;51:25-9.

16 Goodwin JF. Congestive and hypertrophic cardiomyopathies. A decade of study. Lancet 1970;i:731-9.
17 Hamby RI. Primary myocardial disease. A prospective clinical and haemodynamic evaluation in 100 consecutive patients. Medicine (Baltimore) 1970;49: 55-78.

18 Hatle L, Orjavik O, Storstein O. Chronic myocardial disease I. Clinical picture related to long-term prognosis. Acta Med Scand 1976;199:399-405.

19 Shugoll GI, Bowen PJ, Moore JP, Lenkin ML. Followup observations and prognosis in primary myocardial disease. Arch Intern Med 1972;129:67-72.

20 Dye CL, Rosenbaun D, Lowe JC, Behnke RH, Genovese PD. Primary myocardial disease. Part I: clinical features. Ann Intern Med 1963;85:425-41.

21 Greenwood RD, Nadas AS, Fyler DC. The clinical course of primary myocardial disease in infants and children. Am Heart J 1976;92:549-60.

22 Huang SK, Messer JV, Denes P. Significance of ventricular tachycardia in idiopathic dilated cardiomyopathy: observations in 35 patients. Am J Cardiol 1983;51:507-12.

23 Von Olshausen K, Schäfer A, Mehmel HC, Schwarz F, Senges J, Kübler W. Ventricular arrhythmias in idiopathic dilated cardiomyopathy. $\mathrm{Br}$ Heart $J$ 1984;51:195-201.

24 Hatle L, Stake G, Storstein O. Chronic myocardial disease II. Haemodynamic findings related to longterm prognosis. Acta Med Scand 1976;199:407-11.

25 Goodwin JF. Treatment of the cardiomyopathies. Am J Cardiol 1973;32:341-51.

26 Franciosa JA. Effectiveness of long-term vasodilator administration in the treatment of chronic left ventricular failure. Prog Cardiovasc Dis 1982;24:319-30.

27 Franciosa JA. Long-term vasodilator therapy of chronic left ventricular failure: does it work? Int $J$ Cardiol 1984;5:433-9. 